\title{
PRIMER REPORTE DEL ENROLLADOR DE BANDAS OBLICUAS, CHORISTONEURA ROSACEANA (HARRIS) (LEPIDOPTERA: TORTRICIDAE), EN MANZANO EN EL EJIDO VISTA HERMOSA, CIUDAD CUAUHTÉMOC, CHIHUAHUA, MÉXICO
}

Bautista Martínez, N., C. Chavarín Palacio \& E. López Bautista. 2011. First report of oblique
banded leafroller Choristoneura rosaceana (Harris) (Lepidoptera: Tortricidae) in apple in Ejido
Vista Hermosa, Ciudad Cuauhtemoc, Chihuahua, Mexico. Acta Zoológica Mexicana (n. s.), 27(3):
819-824.

ABSTRACT. Choristoneura rosaceana (Harris) is recorded for the first time in apple crops variety 'Golden Delicious' in the Ciudad Cuauhtémoc, Chihuahua, Mexico. Immature stages were described briefly and its damage in foliage and fruits; also the adults main characteristics. The way of arrivalby this pest to the apple crops region was hypothesized.

La producción de manzano en el Norte de México representa una de las actividades frutícolas de mayor importancia agrícola y económica. La producción nacional no es suficiente para abastecer la demanda de esta fruta, por ello es necesario importarla, principalmente de los Estados Unidos de América, así también de Chile, Canadá y Nueva Zelanda (SAGARPA-INCA RURAL 2007). La superficie cultivada de manzana en México hasta el año 2009 fue de 57,000 ha con una producción de 525,000 toneladas (FAOSTAT 2011). Los principales estados productores son Chihuahua, Durango y Coahuila los cuales aportaron $94.3 \%$ de la superficie sembrada y $97 \%$ de la producción nacional (SAGARPA-INCA RURAL 2007). Chihuahua es el estado más importante en cuanto a producción; hasta el 2009 con una superficie cosechada de 22,745.28 ha con una producción de 382,955.20 toneladas (SIAP 2011). El volumen anual promedio de importaciones de manzana ha sido de 135 mil toneladas y Estados Unidos de América es el principal proveedor con una participación del $72 \%$, le siguen en importancia Chile, Canadá y Nueva Zelanda; se estima que el 50\% 


\section{DIRECCIÓN GENERAL DE SANIDAD VEGETAL CENTRO NACIONAL DE REFERENCIA FITOSANITARIA SUBDIRECCION DE DIAGNOSTICO FITOSANITARIO DEPARTAMENTO DE ENTOMOLOGIA Y ACAROLOGIA B00.01.04.01.01:- 03661}

SERVICIO NACIONAL DE SANIDAD, INOCUIDAD Y CALIDAD AGROALIMENTARIA

Dr. Néstor Bautista Martínez Profesor Investigador Titular Colegio de Postgraduados, Campus Montecillo $\mathrm{Km}$. 36.5 Carretera México-Texcoco, Montecillo, Edo. de México. C. P. 56230 Tel. 01 (55) 58-04-59-00 Ext. 1608 y 1607. coorento@colpos.mx

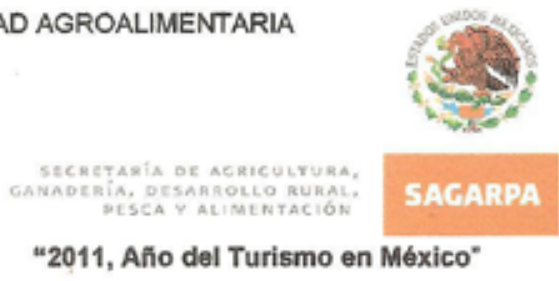

México a D. F., a 13 abril de 2011.

En respuesta a su documento, mediante el cual solicita el posicionamiento oficial para la publicación de una nota cientifica, por la reciente detección de la especie Choristoneura rosaceana (Harris) (Lepidoptera: Tortricidae), conocida como gusano enrollador de las rosáceas, en la zona manzanera del municipio de Ciudad Cuauhtémoc, Chihuahua, durante los meses de julio y agosto de 2010, me permito comunicarie lo siguiente:

Agradecemos, su iniciativa en este tema y a la vez le informo que en este caso en particular, esta Dirección General, no tiene inconveniente en que realice dicha publicación de la nota cientifica, por la detección de la plaga en la zona manzanera de Ciudad Cuauhtémoc, Chihuahua, México, ya que es de interés y de relevancia para esta Dirección General, la información cientifica confiable para el manejo y control de esta plaga.

Por la importancia que representa para esta Dirección General, la regulación y detección oportuna de Choristoneura rosaceana (Harris), considerada como plaga de importancia cuarentenaria en la zona manzanera del municipio de Ciudad Cuauhtémoc, hago de su conocimiento que será necesario, que en la nota que se pretenda publicar se señale claramente los sitios donde se obtuvo las muestras y se haga referencia a su presencia únicamente en este sitio a fin de no generar confusión o interpretaciones erróneas sobre la distribución restringida de esa especie. Asimismo, agradecemos que nos comparta ejemplares para su incorporación a la colección del Centro Nacional de Referencia Filosanitaria, los cuales fueron colectados en la zona afectada y que fueron motivo de este estudio.

Esta Dirección General, podrá revisar la versłón previa a su envió para su publicación, finalmente pongo a su disposición la siguiente dirección electrónica: enrique.vega@senasica.gob.mx, del Jefe de Departamento de Entomologia y Acarologia para que se pueda contactar.

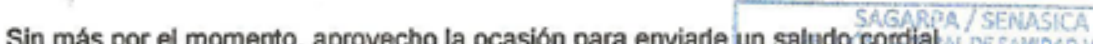

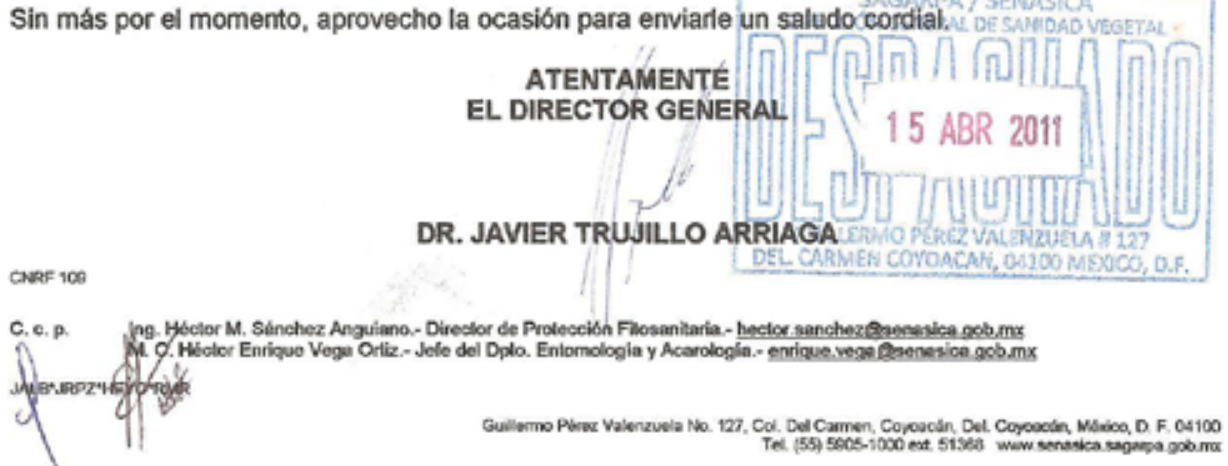


del mercado de calidad selecta se abastece con fruta importada (SAGARPA-INCA RURAL 2007).

El gobierno de México ha publicado una norma oficial para prevenir el ingreso de plagas cuarentenarias del género Choristoneura hacia territorio nacional, regulando la importación de árboles de navidad (NOM-EM-158-SEMARNAT2009). México y los Estados Unidos de América han suscrito un acuerdo para el manejo de las importaciones de cerezas hacia México, en el cual se especifican cuidados para evitar la introducción de algunas plagas tales como Conotrachelus nenuphar, Rhagoletis pomonella, Argyrotaenia citrana y Choristoneura sp. (USDA-APHIS \& SAGARPA 2005). La presencia del enrollador de bandas oblicuas $C$. rosaceana en las zonas templadas de Estados Unidos y Canadá es amplia; la plaga se alimenta de árboles de manzana, principalmente (Hill \& Roelofs 1979). Las limitantes de la producción de manzana en México están relacionadas con aspectos de manejo cultural, nutricional y fitosanitario. En el año 2009 se registraron las primeras observaciones de C. rosacea$n a$ en material procedente de empacadoras de durazno en Casas Grandes, Chihuahua; esta información se dio a conocer por la autoridad fitosanitaria local. En el año 2010, distintos productores reportaron la presencia de una plaga del follaje y fruto de manzana en la región productora de Ciudad Cuauhtémoc, Chihuahua. Por lo anterior, se decidió desarrollar el presente estudio para corroborar o descartar la presencia del enrollador de bandas oblicuas en la zona.

En julio y agosto de 2010 se realizaron colectas de follaje de manzano de tres huertas de aproximadamente 3 ha cada una. Se revisaron 20 árboles al azar en cada huerta; los tres sitios de muestreo se localizaron en dos campos: el campo 15 ( $28^{\circ} 37^{\prime}$ $44.16^{\prime \prime} \mathrm{N}$ y $\left.106^{\circ} 53^{\prime} 16.33^{\prime \prime} \mathrm{O}\right)$ y el campo $18\left(28^{\circ} 45^{\prime} 08.10^{\prime}\right.$ " N y $106^{\circ} 55^{\prime} 57.85^{\prime \prime}$ O), ubicados a una altitud de $2019 \mathrm{msnm}$, pertenecientes al Ejido Vista Hermosa, municipio de Ciudad Cuauhtémoc, Chihuahua. La variedad de los árboles de manzano (Malus domestica) es 'Golden Delicious'.

Las larvas colectadas en el follaje se colocaron en jaulas de madera con cubiertas de tela de organza y se ubicaron a temperatura ambiente entre $18-20{ }^{\circ} \mathrm{C}$ y un periodo de luz de 12:12 h, hasta la emergencia de los adultos. Posteriormente se procedió al montaje y etiquetado de los ejemplares. Las larvas se fijaron en agua caliente (100 ${ }^{\circ} \mathrm{C}$ ) y se pasaron a frascos con alcohol al $70 \%$. Los ejemplares identificados, larvas y adultos, se depositaron en la colección de plagas del Área de Entomología Agrícola del Colegio de Postgraduados, Campus Montecillo, en el Estado de México. En los árboles de manzano se observaron hojas enrolladas y sujetas con seda secretada por las larvas; en este estado, la plaga es de color verde con rayas amarillentas en la parte dorsal de los segmentos corporales. Las larvas colectadas puparon y emergieron 11 palomillas, las cuales se montaron mediante técnicas entomológicas recomendadas para su conservación e identificación mediante caracteres morfológicos de los adultos. La identificación taxonómica de larvas y adultos se realizó por el primer autor 
a

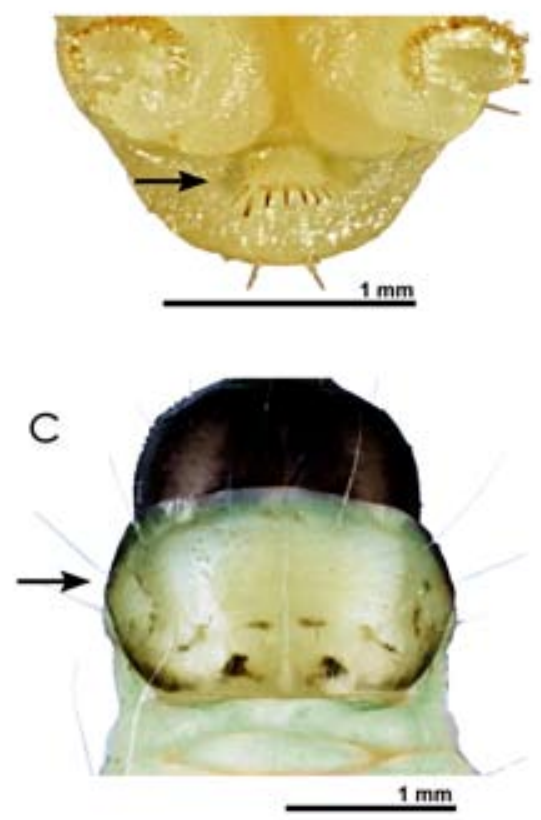

b

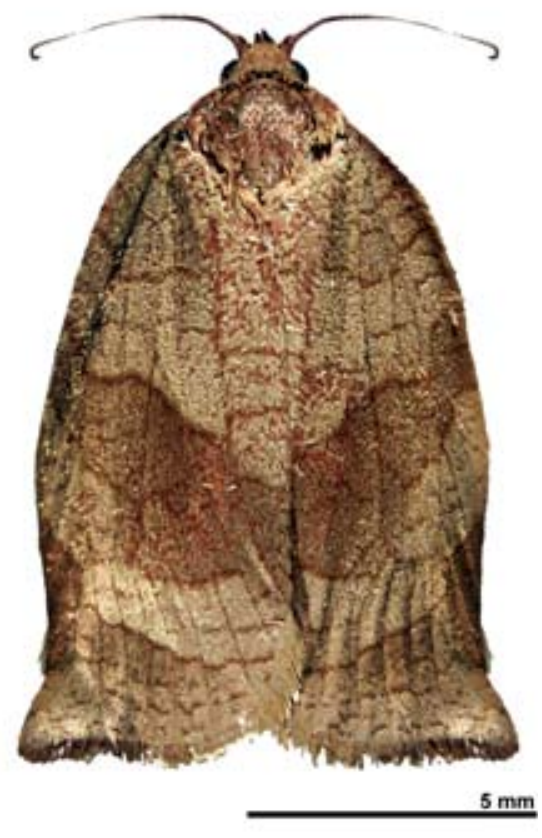

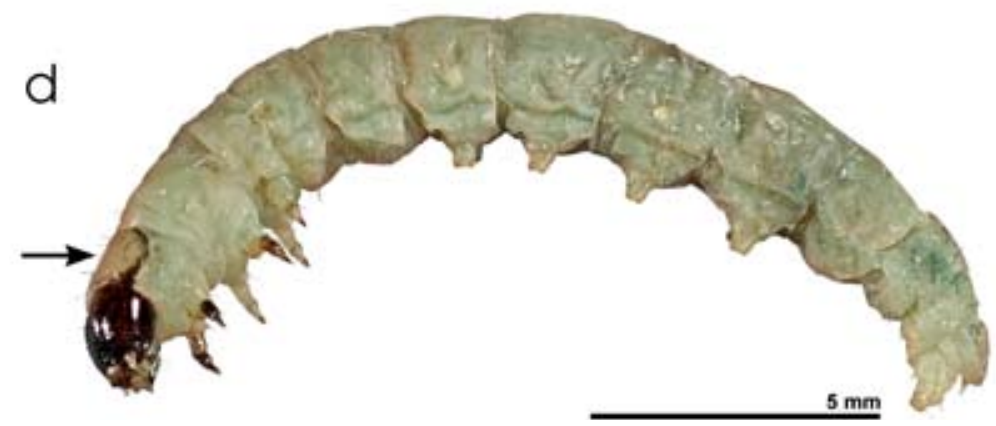

Figura 1. a) Peine anal con 8 dientes; b) adulto con bandas oblicuas de escamas de color café oscuro en las alas anteriores; c) escudo protorácico con coloración clara; d) larva de último estadio.

de esta nota, mediante claves taxonómicas del orden Lepidoptera (MacKay 1962, Brown 1987, Epstein et al. 2004).

La densidad promedio registrada fue de 28 larvas por árbol de manzano, esto con base en observaciones realizadas durante las visitas a los campos de manzano, los 
cuales sumaban 8 hectáreas en total. Las observaciones realizadas indican que la larva de este lepidóptero secreta seda con la que une las hojas de manzano y realiza un nido para refugiarse y pasar al estado de pupa. Se observaron también algunos daños en frutos, cuyos efectos provocan un aspecto corchoso; el porcentaje promedio estimado de frutos dañados fue menor al 1\%. También se observó la presencia de larvas y pupas en el pedúnculo de frutos de manzano. Las características morfológicas estudiadas indicaron que se trata de la especie Choristoneura rosaceana (Harris); para el caso de larvas, resaltó la presencia del peine anal con 8 dientes (Fig. 1a) y el escudo protorácico con una coloración clara en larvas del último estadio (Figs. 1c-1d), lo cual concuerda con las descripciones hechas por MacKay (1962) y Brown (1987); y, en el caso de adultos, la presencia de la banda oblicua de escamas de color café oscuro en las alas anteriores (Fig. 1b), lo cual concuerda con lo indicado por Epstein et al. (2004).

Se sabe que los productores de la región consiguen varetas de manzano en los Estados Unidos de América; por lo anterior, es posible que C. rosaceana haya ingresado a México a través de esta práctica. Considerando la presencia $C$. rosaceana en la región manzanera de Ciudad Cuauhtémoc, Chihuahua, se requiere realizar mayor esfuerzo de investigación en la región para aclarar su estatus fitosanitario, ya que aún es considerada plaga de importancia cuarentenaria y de distribución restringida a esa región, aportando elementos de su impacto económico por el incremento en los costos de producción, evaluar pérdidas de producción, su rango y velocidad de dispersión, hospederos alternos y prácticas de manejo.

\section{LITERATURA CITADA}

Brown, R. L. 1987. Tortricoidea, pp. 419-433. In: F. Stehr (Ed.). Immature Insects. Kendall/Hunt Publ. Co.

Epstein, D., L. Gut \& G. W. Sundin. 2004. A pocket guide for IPM scouting in Michigan apples Michigan State University Extension Bulletin. E-2720 SP.

FAOSTAT. 2011. http://faostat.fao.org (Consultado el 27 de mayo de 2011).

González P. M., N. E. Rocha G., J. Simpson, R. Rodríguez G., J. A. Gallegos I., E. Delgado \& K. Gil. V. 2008. Determinación de algunos atributos de calidad de manzana Starking y Golden Delicious en comparación con sus mutantes y su relación con las unidades frío. Ciencia y Tecnología Alimentaria, 6: 27-32.

Hill, A. S. \& W. L. Roelofs. 1979. Sex pheromone components of the obliquebandedleafroller moth, Choristoneurarosaceana. Journal of Chemical Ecology, 5: 3-11.

MacKay, M. R. 1962. Larvae of the North American Tortricinae (Lepidoptera: Tortricidae). Canadian Entomologist, Supp. 28.

NOM-EM-158-SEMARNAT. 2009. Diario Oficial de la Federación: Noviembre 6 de 2009.

SAGARPA-INCA RURAL. 2007. Sistema Nacional Manzana. Plan Rector. pp. 35-47.

SIAP. 2011. http://www.siap.gob.mx (consultado el 27 de mayo de 2011)

USDA-APHIS-SAGARPA. 2005. Acuerdo Fitosanitario. 4 pp. 
Néstor Bautista Martínez ${ }^{1}$, Claudio Chavarín Palacio ${ }^{1}$ y Everardo López BAUTISTA $^{2}$

${ }^{1}$ Programa de Entomología Agrícola,

Colegio de Postgraduados, Campus Montecillo.

Carr. México-Texcoco, km. 36.5,

C. P. 56230. Montecillo, Estado de México.

$<$ nestor@colpos.mx>

${ }^{2}$ Universidad Autónoma de Sinaloa,

Escuela Superior de Agricultura del Valle del Fuerte.

Juan José Ríos, Sinaloa. 